\title{
Ectrodactyly-ectodermal dysplasia-clefting syndrome with unusual cutaneous vitiligoid and psoriasiform lesions due to a novel single point TP63 gene mutation
}

\author{
Bartłomiej Wawrzycki ${ }^{1}$, Aldona Pietrzak ${ }^{1}$, Grażyna Chodorowska ${ }^{1}$, Agata A. Filip ${ }^{2}$, Veronique Petit ${ }^{1}$, Lidia Rudnicka ${ }^{3}$, \\ Ewa Dybiec $^{4}$, Adriana Rakowska ${ }^{3}$, Agnieszka Sobczyńska-Tomaszewska ${ }^{5}$, Jean Kanitakis ${ }^{6}$
}

\author{
${ }^{1}$ Department of Dermatology, Venereology and Pediatric Dermatology, Medical University of Lublin, Lublin, Poland \\ 2Department of Cancer Genetics, Medical University of Lublin, Lublin, Poland \\ ${ }^{3}$ Department of Dermatology, Medical University of Warsaw, Warsaw, Poland \\ ${ }^{4}$ Department of Pediatric Radiology, Medical University of Lublin, Lublin, Poland \\ ${ }^{5}$ Health Care Center MEDGEN, Warsaw, Poland \\ ${ }^{6}$ Department of Dermatology, Ed. Herriot Hospital, Lyon, France
}

Adv Dermatol Allergol 2019; XXXVI (3): 358-364 DOI: https://doi.org/10.5114/ada.2018.73437

The p63 protein is a member of a highly-conserved family of transcription factors ( $\mathrm{p} 53 / \mathrm{p} 73$ ) which play a pivotal role in ectodermal and epidermal homeostasis as well as orofacial and limb development [1-4]. Novel data suggest its role in the control of senescence, fertility, hearing, neurodevelopment and even cardiogenesis [510]. The gene coding for p63 is located on chromosome $3 q 28$ and consists of 14 exons [11]. It exerts its biologic functions through at least ten different protein isoforms, which are synthesized as a consequence of transcription from alternate promoters (TA and $\Delta \mathrm{N}$ ) and alternative splicing ( $\alpha-, \beta-, \gamma^{-}, \delta$ - and $\varepsilon^{-}$) [12]. The p63 protein is expressed in the nuclei of keratinocytes (predominantly basal ones) of stratified epithelia (skin, tonsils, esophagus and ectocervix), transitional epithelia (urinary tract), simple epithelia (bronchial tree, acini of the breast and prostate, sebaceous and sweat glands), female and male germlines and also to some extent in the germinal centers of lymph nodes $[13,14]$. Investigations on p63-deficient animals and humans bearing mutations in p63 shed light on cutaneous morphogenesis and pathophysiology and revealed p63 as a sentinel of epidermal commitment and differentiation, cell-cell adhesion and basement membrane formation [1, 2].

Heterozygous mutations in TP63 cause seven human developmental syndromes, which include: ADULT syndrome (OMIM 103285); ectrodactyly, ectodermal dysplasia with cleft lip/palate syndrome 3 (OMIM 604292); Hay-Wells syndrome (OMIM 106260); limb-mammary syndrome (OMIM 603543); orofacial cleft 8 (OMIM 129400); Rapp-Hodgkin syndrome (OMIM 129400); and split-hand/foot malformation 4 (OMIM 605289), all of which share at least one common feature [15]. However, the influence of specific mutations or positioning of mutated residues in specific domains on TP63-related pathology remains elusive.

Ectrodactyly, ectodermal dysplasia and cleft lip/palate syndrome 3 (EEC3) belongs to group 1 of ectodermal dysplasias according to a novel clinico-molecular classification [16, 17], which not only denotes involvement of two or more ectodermal derivatives but also encompasses its genetic background and pathogenic mechanism (regulatory changes in transcription and/or expression of genes - in this instance p63). We report here a new patient with EEC3 due to a new heterozygous p63 mutation, and discuss the genotypic-phenotypic correlations of this syndrome.

A 23-year-old man was born to unrelated healthy parents with an unremarkable family history for genetic disorders. Physical examination showed sparse, dry, brittle and slowly growing hair (Figure 1). Most of his nails were dystrophic with longitudinal fissuring and ridging, some also with dorsal pterygium. The patient's skin was fairly pigmented (Fitzpatrick's type 2) and dry but sweating was reportedly normal. Additional cutaneous findings included psoriasiform plaques over both elbows and widespread (neck, chest, abdomen, elbows, antecubital fossae, wrists, knees, dorsal aspects of the hands and feet) hypopigmented, vitiligo-like macules (Figure $1 \mathrm{G}$ ). Histological examination of a psoriasiform cutaneous lesion showed mild psoriasiform epidermal hyperplasia (hyperkeratosis with focal parakeratosis, acanthosis and papillomatosis). The spinous layer contained sparse dyskeratotic keratinocytes (Figure 2 A). PAS

Address for correspondence: Agata A. Filip, Department of Cancer Genetics, Medical University of Lublin, 11 Radziwiłłowska St, 20-080 Lublin, Poland, phone/fax: +48 8144861 00, e-mail: aafilip@hotmail.com Received: 4.01.2018, accepted: 6.01.2018. 
staining was negative. Immunohistochemical staining revealed normal expression of p63 in the epidermis and its adnexa (i.e. nuclear labeling of epidermal keratinocytes with a slightly decreasing intensity from the basal to the granular layer, and nuclear labeling of myoepithelial cells and of basal cells of the dermal eccrine sweat-gland ducts) (Figure 2 C). Ki-67 was expressed by some basal epidermal keratinocytes (Figure 2 D). Filaggrin was normally expressed within the granular layer (Figure 2 B). The skin biopsy taken from a hypopigmented macule showed no noticeable changes on routinely stained sections. Immunohistochemical examination showed a normal expression pattern of the p63 protein (as described above). Immunostaining with antibodies to tyrosinase and MART1/Melan-A showed absence of epidermal melanocytes. S100 protein expression was seen in dendritic cells within the suprabasal epidermal layers (Langerhans cells) but not in the basal cell layer (melanocytes) (data not shown).

Along with skin findings, the patient presented several extracutaneous manifestations. He suffered since childhood from recurrent kerato-conjunctivitis due to right lacrimal duct atresia and complained of nasal bleeding/crusting and oral dryness. ENT examination and sinus $X$-rays showed right maxillary sinus hypoplasia. Oral examination revealed retention of deciduous teeth, hypodontia, misshapen permanent teeth but no lip or palate clefting (Figure $1 \mathrm{~J}$ ). Additional remarkable phenotypic manifestations comprised split-hand and foot deformity of both hands and the right foot as a consequence of syndactyly (left hand and right foot) and ectrodactyly (right hand) (Figures $1 \mathrm{H}-\mathrm{l}$ ).

Finally, several genitourinary problems impaired the quality of life of the patient; they included recur-

A
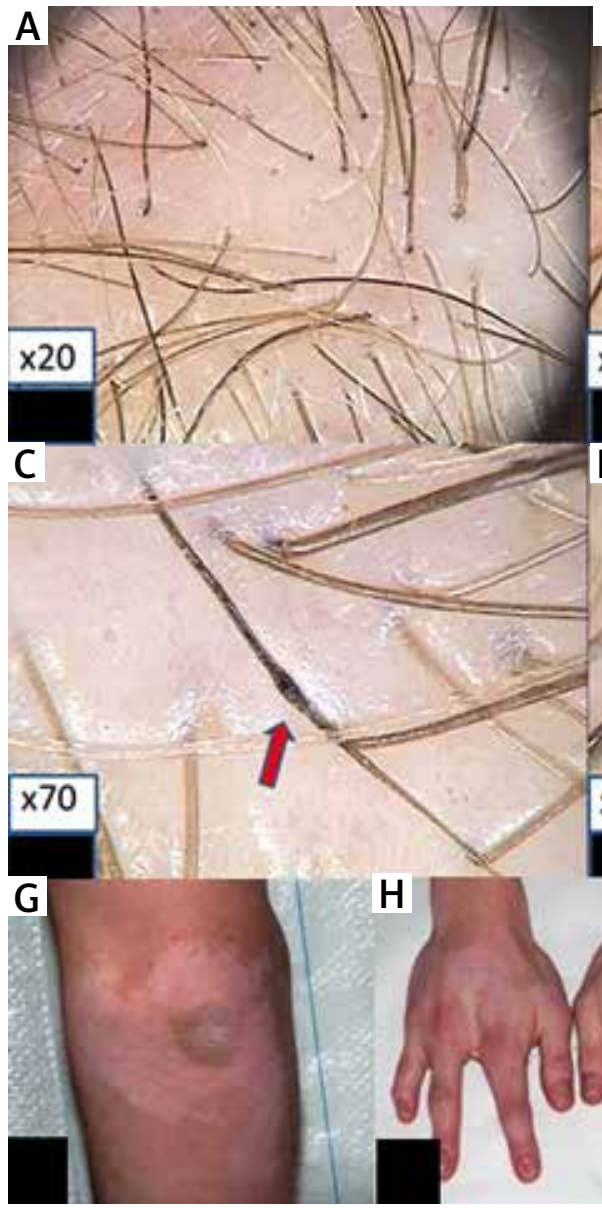

B
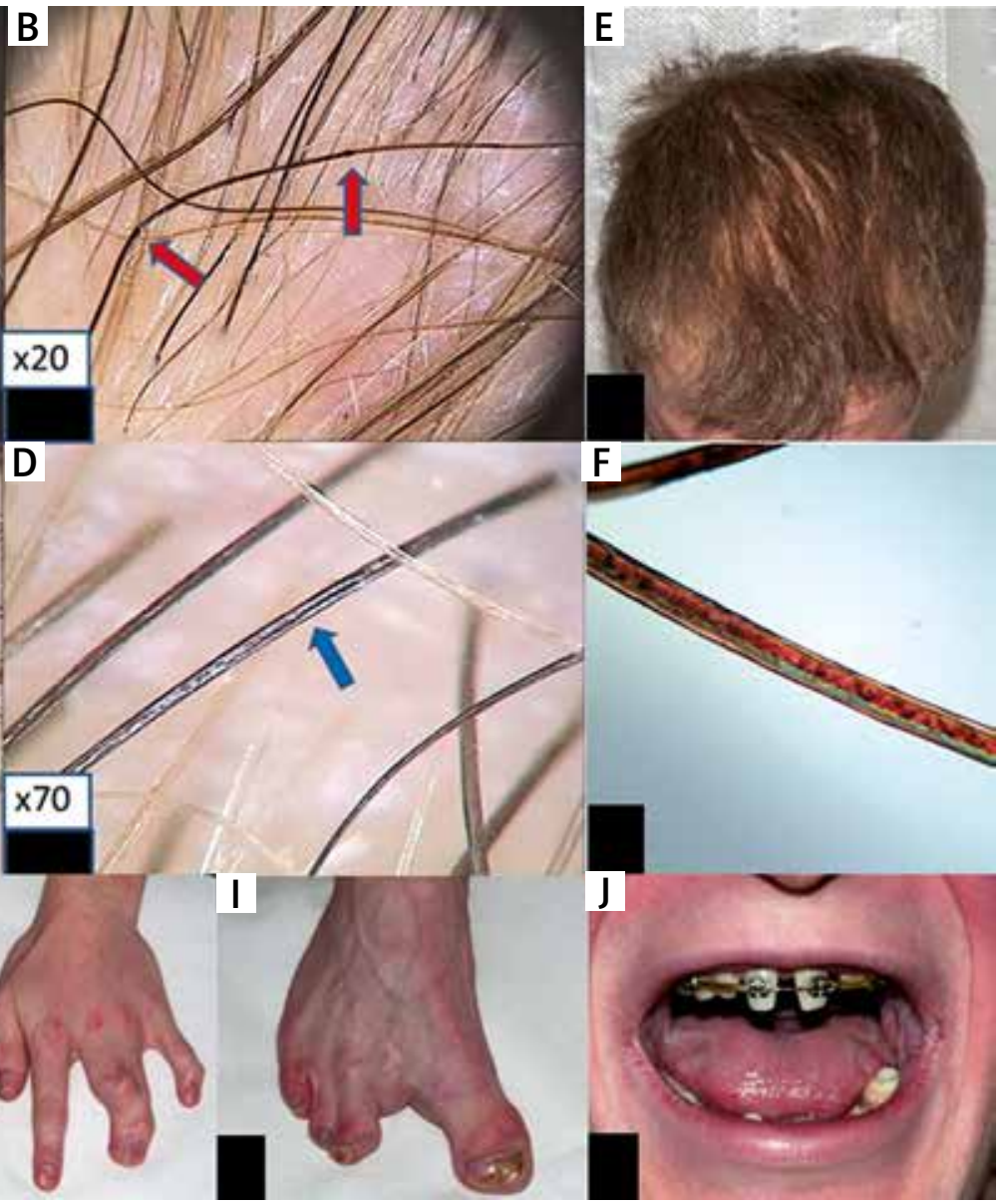

Figure 1. Trichoscopy shows irregular hair-shaft bending at different angles, as typically seen in pili torti (A), irregularly distributed hair-shaft nodosities (B, red arrows) which appear thicker than the normal hair shaft. Internodes are as thick as a normal hair shaft, as seen in pseudomoniletrix (C, red arrows), longitudinal hair-shaft grooving-pili canaliculi (D, blue arrows), and hair color heterogeneity (A, B, C, D), E - hair of the patient, $\mathrm{F}$ - hair shaft microscopic examination with polarized light shows longitudinal grooving and dark and light bands, as seen typically in trichothiodystrophy, $\mathbf{G}$ - psoriasiform hyperplasia and hypopigmented lesions of vitiligo on the left elbow, $\mathbf{H}, \mathbf{I}$ - split hand/foot malformation, $\mathrm{J}$ - teeth during treatment 

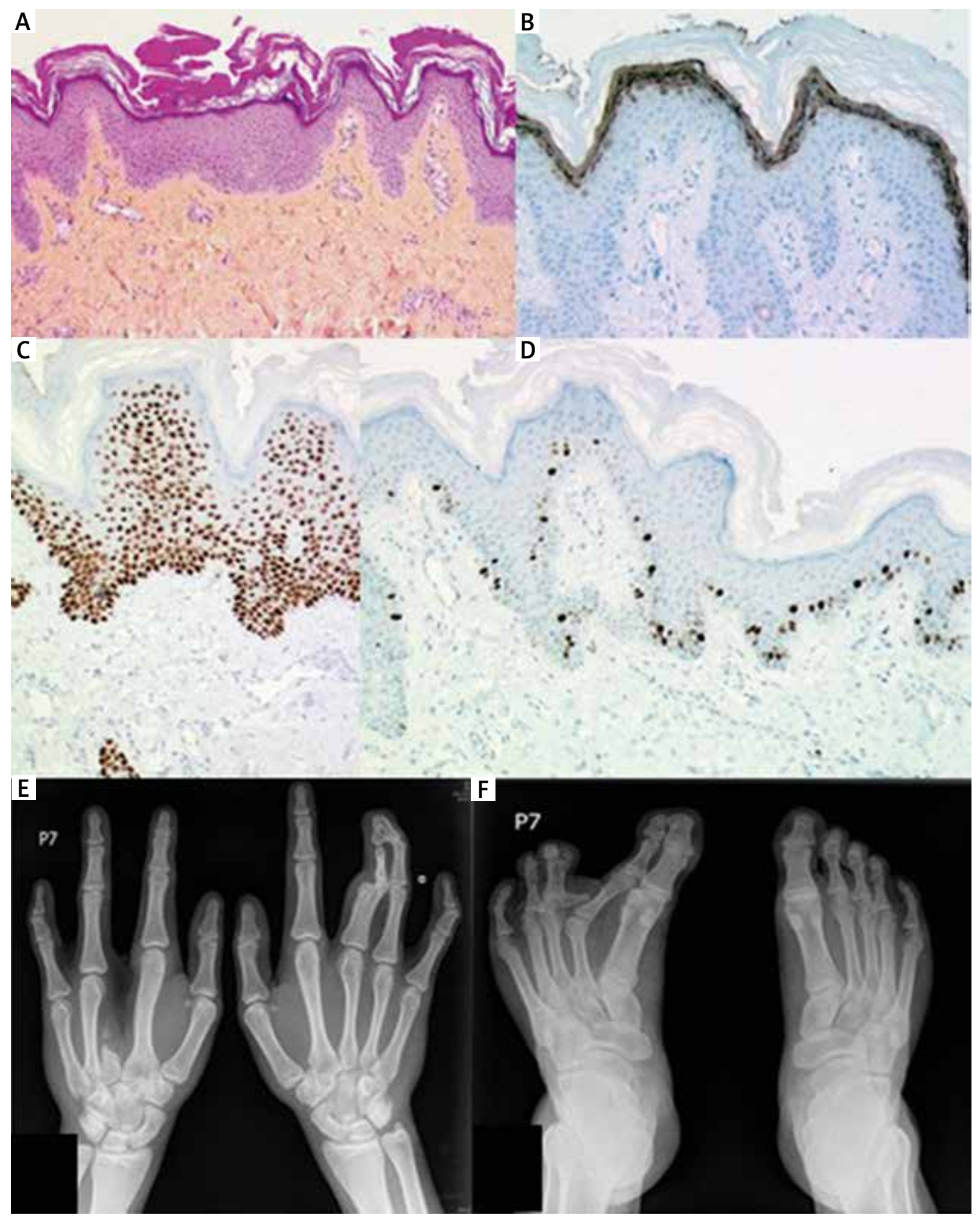

Figure 2. A - Microscopic appearance of a skin biopsy taken from a psoriasiform lesion (hematoxylin-eosin-saffron stain, magnification 100x). B - Filaggrin immunostaining. C - p63 protein expression. D - MIB-1/Ki67 expression (magnification 250x). Radiological examination of hands and feet shows split hand (E) and foot (F) malformation as a consequence of both ectrodactyly and syndactyly 
rent cystitis, hematuria and hypospadias. Abdominal ultrasonography (USG) showed a hypoplastic left kidney and an hyperechogenic, inhomogeneous right kidney. Cystoscopy performed on several occasions revealed hyperemia and exaggerated bladder trabeculae. Renal SPECT tomography and scintigraphy revealed dilatation of the right pyelocalyceal system, interstitial alterations and cortical thinning of the upper segment of the right kidney, moderately impaired glomerular filtration rate, decreased urinary outflow, urinary reflux, urine retention after micturition in the whole kidney (especially in the pyelocalyceal system) and dilatation of the right ureter. Global glomerular filtration rate (GFR) was estimated to $93.1 \mathrm{ml} / \mathrm{min}$ - individual values for left and right kidney were 17.9 and $75.2 \mathrm{ml} / \mathrm{min}$ respectively.

Skeletal X-rays showed: 1) right hand: ectrodactyly of digit III (lack of phalanges, diaphysis and distal epiphysis of metacarpal bone III); visible epiphysis and small osseous fragment of proximal metaphysis of metacarpal bone III; within soft tissue, two small osseous fragments between metacarpal bones II and IV; 2) left hand: syndactyly (soft tissue) of digits III and IV; deformity of distal part of diaphysis and epiphyseal-metaphyseal segment of proximal phalanx III; synostosis within the proximal interphalangeal joint of digit III; deformity of distal phalanges of digits III and IV; synostosis of distal phalanges of digits III-IV; synostosis within the distal interphalangeal joint of digit IV; 3) right foot: an additional, horizontal arrangement, residual middle phalanx of digit III, connected by additional joint surface to the metatarsophalangeal joint; deformity of the distal phalanges of digits IV and V; syndactyly of digits III-IV and IV-V. Both feet showed shortening of the middle phalanx of digit $V$ with abnormal position of the joint surfaces of the distal interphalangeal joints (Figures $2 \mathrm{E}, \mathrm{F}$ ).

With informed consent, in accordance with the Declaration of Helsinki and approval from the Medical University Bioethics Committee (approvals no: KE -0254/182/2015 and KE -0254/229/2016), peripheral blood was obtained from the patient and his healthy sister with the purpose of DNA isolation. Molecular analysis of the TP63 gene using direct DNA sequencing showed the missense mutation p.G314E (NP_003713.3: p.Gly314Glu, NM_003722.4: c.941G>A) (Figure 3). This mutation is not yet registered in any database, including HGMD (the Human Gene Mutation Database). It involves a highly conserved nucleotide and amino acid. Bioinformatics analysis using SIFT and MutationTaster software showed the pathogenic nature of the identified mutation. The clinical evaluation of the patient and the results of the genetic testing confirmed the diagnosis of EEC3.

The EEC syndrome, a member of a larger family of p63-related disorders, comprises three cardinal features: ectrodactyly (absence of the central parts of the hands and feet, also known as split-hand/foot malformation), ectodermal dysplasia, and cleft lip (with or without cleft palate). Not all patients present with the complete triad of these main signs, and the severity of each feature varies among affected individuals [18-24].

Our patient presented the typical constellation of findings of EEC, comprising skin, hair and nail involvement, lacrimal duct obstruction, dental and skeletal malformations, genitourinary malformations and symptoms and oral and nasal dryness, probably due to impaired structure/function of mucous and salivary glands in the setting of ectodermal dysplasia [25].

Hypopigmented and hyperkeratotic-scaly lesions of the skin have been previously reported in EEC patients [26]. In our patient, microscopic examination of the erythematous-scaly lesion showed psoriasiform changes, whereas the hypopigmented macules showed absence of melanocytes, consistent with vitiligo. Despite the fact that a TP63 mutation was present in this patient, the expression of 663 in his skin appeared normal, likely because the epitope recognized by the anti-p63 antibody we used (clone 4A4) was unaffected by the mutation.

The normal Ki-67 and $\mathrm{p} 63$ protein expression in the lesional skin of our patient is consistent with previous observations showing that the p63 protein is expressed in the skin despite the presence of a mutation [27-29]. Molecular alterations probably result in reduced transcriptional activity of the impaired p63 on several gene promoters [30-35]. Regarding filaggrin, this was normally expressed in the skin of our patient. Candi et al. [36] found that p63-/- animals had decreased, but still measurable, filaggrin (along with K14 and loricrin) expression, suggesting that their production is regulated by genes other than TP63.

Although genitourinary abnormalities are not common (or often overlooked) in EEC, they may cause nonnegligible morbidity. The most commonly reported abnormalities include absent or hypoplastic kidneys, hydronephrosis, hydro-ureter, duplication of the urinary collection system, hypospadias and cryptorchidism [3740]. Many patients complain of micturition problems and recurrent cystitis. Bladder biopsies show thin, atrophic or dysplastic bladder epithelium, or interstitial cystitis $[39,40]$. Our patient had both structural and functional disturbances, possibly reflecting interrupted epithelialmesenchymal interactions by the mutant p63 [41-43].

Analysis of genotype-phenotype correlation in 152 patients with p63-associated EEC revealed hair, teeth, nail and lacrimal duct involvement in about half of them [19]. Cleft lip/palate was present in $39 \%$ and $40 \%$ of cases respectively, ectrodactyly in 68\% (43\% also had syndactyly), skin involvement in 34\%, hypohidrosis in $11 \%$ and urinary tract problems in 15\%. Mammary gland/nipple hypoplasia and hearing impairment were found in $11 \%$ and $7 \%$ of patients, respectively. The variable penetrance (the average incidence of any cardinal sign does not exceed $50 \%$ ) seen in EEC syndrome can be partially explained by the specific effects of different mutations. Five argi- 


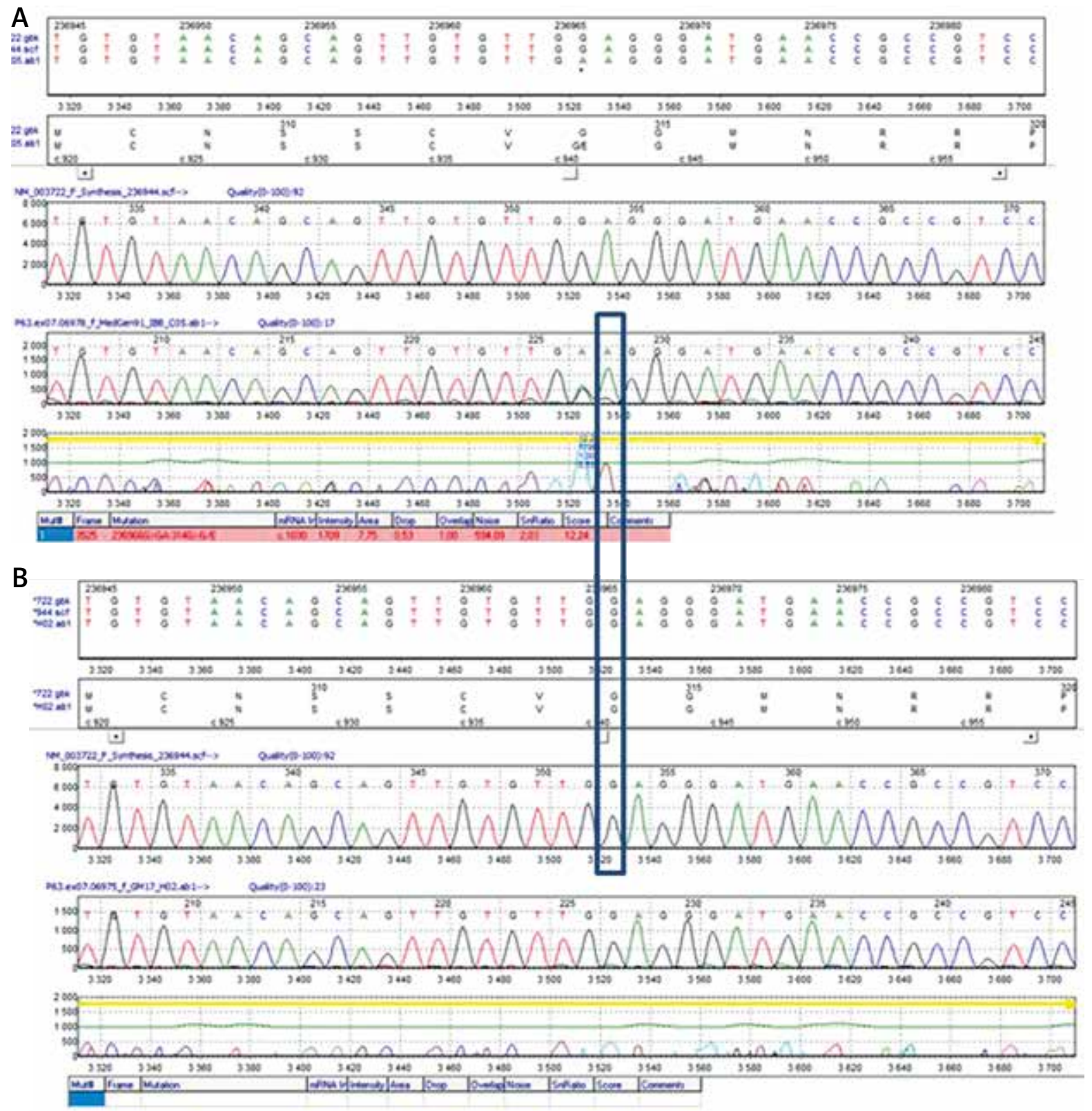

Figure 3. Electropherogram of sequencing results of proband's DNA (A) and his healthy sister (B). The site of the substitution is indicated by a frame

nine hotspot mutations (according to the novel HGVS nomenclature: p.Arg243Trp, p.Arg266Gln, p.Arg318His/ Cys/Ser, p.Arg319His/Cys/Ser, p.Arg343Trp/Pro) located in the DNA-binding domain (DBD) region of the p63 protein account for almost $90 \%$ of all known mutations [21]. For instance, patients bearing the p.Arg243Trp mutation usually present with typical EEC findings, but have a lower incidence of orofacial clefting (26\% vs. $40 \%$ ) and hypohidrosis; those with the p.Arg227GIn mutation are less frequently affected by orofacial clefting (cleft lip 0\%, cleft palate $7 \%$ ) and limb defects (40\% vs. 68\%), but have a higher risk of genitourinary complications (39\%). Conversely, the p.Arg304Trp/Pro mutation is associated with orofacial clefting in $80 \%$ of cases, whereas this reaches on average $40 \%$.

Our patient had a new p.Gly314Glu heterozygous mutation of the TP63 gene, yet with signs and symptoms comparable to the typical clinical presentation, with the closest clinical similarities to individuals with p.Arg243Trp R204 and p.Arg266Gln R227 mutations.

We can also presume that cutaneous, craniofacial, long-bone and genitourinary symptoms depend partly on 
p63-response elements. BMP7, FGFR2B, JAG1, NOTCH1, Bcatenin, EDAR, P-cadherin, DLX5, DLX6, and IRF6 are only some of up to 328 genes whose expression is altered in mutant EEC keratinocytes [2, 33-35]. It was shown recently that the two transcription factors p63 and BRG1 form a complex network of reciprocal interactions controlling remodeling of the higher-order chromatin structure of the epidermal differentiation complex locus [44] and the urothelial cell fate during ureter development [42].

Establishing strict genotype-phenotype correlations is complicated for several reasons, including varying expression and ability of each p63 isoform to transactivate or repress p63-dependent genes, genetic background, epigenetic modifications, the impact of mutant/ wild type ratio on organ involvement and subtle genetic alterations with a striking phenotype shift from one side and the same mutation producing distinct manifestations on the other [31-33, 36, 37]. For instance, recent research on genetically engineered mouse models of EEC syndrome found that animals heterozygous for Trp63 encoding the same single amino acid mutation, which differed only in the presence or absence of the neomycin cassette within intron 4, displayed marked heterogeneity regarding development, penetrance and symptom severity [32]. Furthermore, based on the same allelic series of mouse EEC model, it was found that (contrasting with clefting and skin pathology, which reflected loss of Trp63 function), limb anomalies were due to gain and/or dominant-negative effects of Trp63. Moreover the TA-p63 isoform was found to be an important factor modulating EEC phenotype penetrance and expressivity regarding all three cardinal EEC features [32].

In conclusion, the molecular basis of EEC seems complex. Our patient carried a new p.Gly314Glu heterozygous mutation of the $p 63$ gene that affects the sequence of exon 7, which encodes the DBD (amino acid residues 123-340 of the p63 protein) [45]. The glycine at position 314 is conserved in two other TP53 gene family members, i.e. TP53 and TP73 (at positions 243 and 264, respectively). It does not directly contact DNA; however, prediction of DNA-protein interaction in the $\mathrm{p} 63$ protein model proposed by Celli et al. [46] has shown that mutations within this region might alter the positioning of the p63binding surface; this in turn may result in diminishing the DNA-binding capacity of p63 and abolishing its activity as a transcription factor $[46,47]$.

The location of the mutation site influences the clinical presentation of the disease. Studies of genotype/phenotype correlations in EEC are additionally hindered by its reduced penetration and variable expressivity, which accounts for phenotypic variability in the affected members of the same family. The identification of the novel p.Gly314Glu mutation further expands the knowledge concerning the causes of EEC, and confirms the necessity of sequencing the TP63 gene (primarily exons 4-8) in patients presenting with symptoms, in order to identify the leading cause.

\section{Acknowledgments}

Grant of Medical University of Lublin (Poland), DS 168 and DS 164.

\section{Conflict of interest}

The authors declare no conflict of interest.

\section{References}

1. Vanbokhoven H, Melino G, Candi E, Declercq W. p63, a story of mice and men. J Invest Dermatol 2011; 131: 1196-207.

2. Koster MI. p63 in skin development and ectodermal dysplasias. J Invest Dermatol 2010; 130: 2352-8.

3. Lu Y, Abbassi S, Li F, et al. Distinct function of P63 isoforms during embryonic skeletal development. Gene 2013; 519: 251-9.

4. Leslie EJ, Marazita ML. Genetics of cleft lip and cleft palate. Am J Med Genet Part C Semin Med Genet 2013; 163C: 246-58.

5. Keyes WM, Mills AA. p63: a new link between senescence and aging. Cell Cycle 2006; 5: 260-5.

6. Amelio I, Grespi F, Annicchiarico-Petruzzelli M, Melino G. p63 the guardian of human reproduction. Cell Cycle 2012; 11: 4545-51.

7. Cancino GI, Yiu AP, Fatt MP, et al. p63 Regulates adult neural precursor and newly born neuron survival to control hippocampal-dependent behavior. J Neurosci 2013; 33: 12569-85.

8. Terrinoni A, Serra V, Bruno E, et al. Role of p63 and the Notch pathway in cochlea development and sensorineural deafness. Proc Natl Acad Sci USA 2013; 110: 7300-5.

9. Wolchinsky Z, Shivtiel S, Kouwenhoven EN, et al. Angiomodulin is required for cardiogenesis of embryonic stem cells and is maintained by a feedback loop network of p63 and Activin-A. Stem Cell Res 2014; 12: 49-59.

10. Paris M, Rouleau M, Pucéat M, Aberdam D. Regulation of skin aging and heart development by TAp63. Cell Death Differ 2012; 19: 186-93.

11. Mills AA. p63: oncogene or tumor suppressor? Curr Opin Genet Dev 2006; 16: 38-44.

12. Mangiulli M, Valletti A, Caratozzolo MF, et al. Identification and functional characterization of two new transcriptional variants of the human p63 gene. Nucleic Acids Res 2009; 37: 6092-104.

13. Di Como CJ, Urist MJ, Babayan I, et al. p63 expression profiles in human normal and tumor tissues. Clin Cancer Res 2002; 8: 494-501.

14. Nylander K, Vojtesek B, Nenutil R, et al. Differential expression of p63 isoforms in normal tissues and neoplastic cells. J Pathol 2002; 198: 417-27.

15. Online Mendelian Inheritance in Man, $\mathrm{OMIM}^{\circledR}$. Johns Hopkins University, Baltimore, MD. MIM Number: $\{604292\}$ : \{1/5/2012\}: World Wide Web URL: http://omim.org/.

16. Priolo M. Ectodermal dysplasias: an overview and update of clinical and molecular-functional mechanisms. Am J Med Genet A 2009; 149A: 2003-13.

17. García-Martín P, Hernández-Martín A, Torrelo A. Ectodermal dysplasias: a clinical and molecular review. Actas Dermosifiliogr 2013; 104: 451-70. 
18. Itin PH. Etiology and pathogenesis of ectodermal dysplasias. Am J Med Genet A 2014; 164A: 2472-7.

19. Rinne T, Hamel B, van Bokhoven H, Brunner HG. Pattern of p63 mutations and their phenotypes - update. Am J Med Genet A 2006; 140: 1396-406.

20. Brunner HG, Hamel BC, Van Bokhoven H. The p63 gene in EEC and other syndromes. J Med Genet 2002; 39: 377-81.

21. Rinne T, Brunner HG, van Bokhoven H. p63-associated disorders. Cell Cycle 2007; 6: 262-8.

22. Buss PW, Hughes HE, Clarke A. Twenty-four cases of the EEC syndrome: clinical presentation and management. J Med Genet 1995; 32: 716-23.

23. Clements SE, Techanukul T, Coman D, et al. Molecular basis of EEC (ectrodactyly, ectodermal dysplasia, clefting) syndrome: five new mutations in the DNA-binding domain of the TP63 gene and genotype-phenotype correlation. $\mathrm{Br}$ J Dermatol 2010; 162: 201-7.

24. Bigatà X, Bielsa I, Artigas M, et al. The ectrodactyly-ectodermal dysplasia-clefting syndrome (EEC): report of five cases. Pediatr Dermatol 2003; 20: 113-8.

25. Itin PH, Fistarol SK. Ectodermal dysplasias. Am J Med Genet Part C (Semin Med Genet) 2004; 131C: 45-51.

26. Knaudt B, Volz T, Krug M, et al. Skin symptoms in four ectodermal dysplasia syndromes including two case reports of Rapp-Hodgkin-Syndrome. Eur J Dermatol 2012; 22: 605-13.

27. Kim SY, Cho HJ, Kim DS, et al. Differential expression of p63 isoforms in normal skin and hyperproliferative conditions. J Cutan Pathol 2009; 36: 825-30.

28. Shen CS, Tsuda T, Fushiki S, et al. The expression of p63 during epidermal remodeling in psoriasis. J Dermatol 2005; 32: 236-42.

29. Browne G, Cipollone R, Lena AM, et al. Differential altered stability and transcriptional activity of Np63 mutants in distinct ectodermal dysplasias. J Cell Sci 2011; 124: 2200-7.

30. Gu X, Lundqvist EN, Coates PJ, et al. Dysregulation of TAp63 mRNA and protein levels in psoriasis. J Invest Dermatol 2006; 126: 137-41.

31. Monti P, Russo D, Bocciardi R, et al. EEC-and ADULT-associated TP63 mutations exhibit functional heterogeneity toward P63 responsive sequences. Hum Mutat 2013; 34: 894-904.

32. Vernersson Lindahl E, Garcia EL, Mills AA. An allelic series of Trp63 mutations defines TAp63 as a modifier of EEC syndrome. Am J Med Genet A 2013; 161A: 1961-71.

33. Laurikkala J, Mikkola ML, James M, et al. p63 regulates multiple signaling pathways required for ectodermal organogenesis and differentiation. Development 2006; 133: 1553-63.

34. Pozzi S, Zambelli F, Merico D, et al. Transcriptional network of p63 in human keratinocytes. PLoS One 2009; 4: e5008.

35. Shen J, van den Bogaard EH, Kouwenhoven EN, et al. APR246/PRIMA-1(MET) rescues epidermal differentiation in skin keratinocytes derived from EEC syndrome patients with p63 mutations. Proc Natl Acad Sci USA 2013; 110: 2157-62.

36. Candi E, Rufini A, Terrinoni A, et al. Differential roles of p63 isoforms in epidermal development: selective genetic complementation in p63 null mice. Cell Death Differ 2006; 13 1037-47.

37. King KE, Ponnamperuma RM, Gerdes MJ, et al. Unique domain functions of p63 isotypes that differentially regulate distinct aspects of epidermal homeostasis. Carcinogenesis 2006; 27: 53-63.

38. Maas SM, de Jong TP, Buss P, Hennekam RC. EEC syndrome and genitourinary anomalies: an update. Am J Med Genet 1996; 63: 472-8.
39. Maclean K, Holme SA, Gilmour E, et al. EEC syndrome, Arg227GIn TP63 mutation and micturition difficulties: is there a genotype-phenotype correlation? Am J Med Genet A 2007; 143A: 1114-9.

40. Sripathomsawat W, Tanpaiboon P, Heering J, et al. Phenotypic analysis of Arg227 mutations of TP63 with emphasis on dental phenotype and micturition difficulties in EEC syndrome. Am J Med Genet A 2011; 155A: 228-32.

41. Zhang Y, Yan W, Chen X. P63 regulates tubular formation via epithelial-to-mesenchymal transition. Oncogene 2014; 33: 1548-57.

42. Weiss RM, Guo S, Shan A, et al. Brg1 determines urothelial cell fate during ureter development. I Am Soc Nephrol 2013; 24: 618-26.

43. Ching BJ, Wittler L, Proske J, et al. p63 (TP73L) a key player in embryonic urogenital development with significant dysregulation in human bladder exstrophy tissue. Int J Mol Med 2010; 26: 861-7.

44. Mardaryev AN, Gdula MR, Yarker JL, et al. p63 and Brg1 control developmentally regulated higher-order chromatin remodelling at the epidermal differentiation complex locus in epidermal progenitor cells. Development 2014; 141: 101-11.

45. Ianakiev P, Kilpatrick MW, Toudjarska I, et al. Split-hand/ split-foot malformation is caused by mutations in the $\mathrm{p} 63$ gene on 3q27. Am J Hum Genet 2000; 67: 59-66.

46. Celli J, Duijf P, Hamel BC, et al. Heterozygous germline mutations in the p53 homolog p63 are the cause of EEC syndrome. Cell 1999 Oct 15; 99: 143-53.

47. Cho Y, Gorina S, Jeffrey PD, Pavletich NP. Crystal structure of a p53 tumor suppressor-DNA complex: understanding tumorigenic mutations. Science 1994; 265: 346-55. 